\title{
The incidence and risk factors of stroke in patients with acute myocardial infarction treated invasively and concomitant impaired renal function
}

\author{
Tomasz Podolecki ${ }^{1}$, Radosław Lenarczyk ${ }^{1}$, Jacek Kowalczyk ${ }^{1}$, \\ Andrzej Świątkowski ${ }^{1}$, Piotr Chodór ${ }^{1}$, Patrycja Pruszkowska-Skrzep ${ }^{1}$, \\ Agnieszka Sędkowska ${ }^{1}$, Lech Poloński², Zbigniew Kalarus ${ }^{1}$ \\ ${ }^{1}$ Department of Cardiology, Congenital Heart Diseases and Electrotherapy, \\ Silesian Medical University, Silesian Center of Heart Diseases, Zabrze, Poland \\ ${ }^{2} 3^{\text {rd }}$ Department of Cardiology, Silesian Medical University, \\ Silesian Center of Heart Diseases, Zabrze, Poland
}

\begin{abstract}
Background: Impaired renal function is a marker of poor prognosis in patients with acute myocardial infarction (AMI). The aim of the study was to assess the incidence and independent predictors of stroke in population of patients with AMI treated invasively and concomitant impaired renal function (IRF).

Methods: We analyzed 2,520 consecutive AMI patients admitted to our Center between 2003 and 2007 and treated with percutaneous coronary intervention. The whole population was divided into patients with IRF, defined as glomerular filtration rate $<60 \mathrm{~mL} / \mathrm{min} / 1.73 \mathrm{~m}^{2}$ or contrast induced nephropathy (IRF group, $n=933$; 37.02\%) and patients without IRF (control group, $n=1587 ; 62.98 \%$ ). The IRF group was subjected to further analysis. Data on long-term follow-up were screened to identify the patients who experienced stroke.
\end{abstract}

Results: During median of 25.5 months of follow-up 52 (2.07\%) the patients experienced stroke - 33 (3.54\%) in the IRF group and 19 (1.2\%) patients in the control group. The risk of major adverse cardiovascular events in the IRF group, including repeated AMI (68.8 vs. $14.9 \%, p<0.001)$ and death $(45.5 \mathrm{vs} .25 .1 \%, p<0.05)$ was significantly higher in patients with stroke. Previous stroke (HR 6.85), female gender (HR 3.13), as well as STEMI anterior (HR 2.55) were independent risk factors of stroke in this population.

Conclusions: Patients with AMI treated invasively and concomitant IRF were at higher risk of stroke occurrence in the future. Stroke was associated with poor outcome in the studied population. Independent predictors of stroke in patients with IRF and AMI treated invasively were different from commonly recognized stroke predictors. (Cardiol J 2013; 20, 6: 672-678)

Key words: stroke, impaired renal failure, myocardial infarction, percutaneous coronary intervention, contrast-induced nephropathy

Address for correspondence: Tomasz Podolecki, MD, Department of Cardiology, Congenital Heart Diseases and Electrotherapy, Silesian Medical University, Silesian Center for Heart Diseases, ul. Szpitalna 2, 41-800 Zabrze, Poland, tel: +483237336 82, fax: +4832373 37 92, e-mail: tomekpod@interia.pl

Received: 27.02.2013 Accepted: 07.04.2013 


\section{Introduction}

Impaired renal function (IRF) is a well recognized marker of poor prognosis in general population, as well as in patients after acute myocardial infarction (AMI) [1-3]. Disturbed renal function is associated with both higher mortality and higher risk of other adverse cardiac events (MACE), including stroke [3-5]. The incidence of stroke among AMI survivors may approach $1.2 \%$ within 30 -day observation and more than $2 \%$ during 12 -month observation [6, 7]. According to Mielniczuk et al. [8], the risk of stroke is over 2-fold higher in AMI patients with concomitant IRF. Based on previously published studies, atrial fibrillation (AF), diabetes mellitus, previous stroke, advanced age, female gender and anterior infarction were recognized as stroke predictors in patients after AMI [6, 7, 9, 10]. However, none of the above-mentioned studies was based on a homogenous group of consecutive AMI patients treated with percutaneous coronary intervention (PCI).

The aim of the study was to assess the incidence and independent predictors of stroke in patients with AMI treated invasively and burdened with disturbed renal function.

\section{Methods}

The analysis was conducted on the database of 2,520 consecutive AMI patients admitted to our Center between 2003 and 2007. All the patients had coronary angiography and subsequent PCI performed without any delay. The aim of the procedure was to restore TIMI grade 3 flow, with remaining residual stenosis of less than $30 \%$, in the infarct-related artery (IRA). A single dose of oral aspirin (300-500 mg) and 5,000-10,000 U of intravenous heparin were administered to all the patients before coronary angiography, whereas a loading dose of Clopidogrel was given before stent implantation. If necessary, additional doses of heparin were administered, according to the activated clotting time. The duration of dual anti-platelet therapy, as well as concomitant post-myocardial treatment were in line with current recommendations of cardiac societies [11-13].

Data concerning demographic, clinical, laboratory, echocardiographic and angiographic characteristics were obtained from a single-center prospective cohort study of acute coronary syndromes. Data on remote follow-up were obtained from the insurer - the National Health Fund. The diagnosis and type of stroke in the post-AMI period were established based on the international classification of diseases (ICD-10) codes once all the follow-up data had been collected and analyzed. The type of stroke (ischemic, hemorrhagic or undetermined) was additionally confirmed by a review of hospital charts obtained from hospitals where patients with stroke had been admitted to. The whole AMI population was divided into patients with (IRF group, $\mathrm{n}=933 ; 37.02 \%$ ) and without IRF (control group, $\mathrm{n}=1587 ; 62.98 \%$ ). Subsequently the IRF group was subdivided into patients with stroke $(\mathrm{n}=33 ; 3.54 \%)$ and free of stroke $(\mathrm{n}=900$; $96.46 \%)$ during remote observation. All the data regarding long-term outcomes were reviewed to ascertain that they fulfill the predefined criteria of adverse cardiovascular (CV) events.

The study was approved by the local authorities and all patients were asked for written informed consent.

\section{Definitions}

Renal function was assessed with estimated glomerular filtration rate (GFR) calculated by means of the simplified Modification of Diet in Renal Disease formula [14].

Contrast induced nephropathy (CIN) was defined as an elevation of serum creatinine by at least $44.2 \mu \mathrm{mol} / \mathrm{L}(0.5 \mathrm{mg} / \mathrm{dL})$ or a $25 \%$ increase from the baseline value within $48 \mathrm{~h}$ after contrast exposure.

IRF was defined as GFR less than $60 \mathrm{~mL} /$ $/ \mathrm{min} / 1.73 \mathrm{~m}^{2}$ and/or CIN during index hospitalization.

Stroke was recognized if the development of an acute focal neurological deficit with a duration of more than $24 \mathrm{~h}$ was documented.

Hemorrhagic stroke was diagnosed if symptoms/signs were associated with bleeding on the computed tomography (CT) scan, while ischemic stroke was recognized if there was no evidence of bleeding on the CT scan. Undetermined stroke was diagnosed when no CT scan was performed.

MACE was defined as a composite of death, non-fatal MI and repeated percutaneous or surgical coronary revascularization.

\section{Statistics analysis}

Continuous parameters were expressed as mean \pm standard deviation, whilst categorical variables as numbers and percentages. Comparative analysis between the groups was performed with the Student's t-test for continuous variables and $\chi^{2}$ or Fisher's exact test, as appropriate, for dichotomous parameters. Independent stroke predictors were identified with the multivariate Cox regression model and expressed as hazard ratio (HR) 
with 95\% confidence interval (CI). Two Cox-proportional hazards models were created to identify the most powerful set of independent risk factors. All parameters which differed significantly between the stroke and the non-stroke groups were considered covariates in the first regression model. The second model additionally incorporated factors commonly believed to influence the risk of stroke: diabetes mellitus, arterial hypertension, AF, left ventricular ejection fraction (LVEF) $<35 \%$, age $>65$ and the use of vitamin K antagonists (VKA). The more predictive Cox regression model (with higher $\chi^{2}$ value) was subjected to further analysis. Cumulative proportions of patients free of stroke in every group were plotted as Kaplan-Meyer survival curves and compared with logrank tests between different categories. $\mathrm{P}$ value $<0.05$ was considered statistically significant. The software package Statistica (version 6.0, StatSoft Inc., Tulsa, OK, USA) was used for statistical analysis.

\section{Results}

\section{Stroke incidence}

Among 933 patients with impaired renal function $293(31.4 \%)$ had GFR less than $60 \mathrm{~mL} /$ $/ \mathrm{min} / 1.73 \mathrm{~m}^{2}, 466$ (49.95\%) developed CIN and $174(18.65 \%)$ patients had both GFR $<60 \mathrm{~mL} /$ $/ \mathrm{min} / 1.73 \mathrm{~m}^{2}$ and CIN during index hospitalization. During median observation of 25.5 (range: 0-57) months 33 patients $(3.54 \%)$ experienced stroke in the IRF group and only $19(1.2 \%)$ in the control group $(\mathrm{p}<0.001)$. Patients with GFR less than $60 \mathrm{~mL} / \mathrm{min} / 1.73 \mathrm{~m}^{2}$ additionally complicated by CIN had double as high risk of stroke as subjects with GFR $<60 \mathrm{~mL} / \mathrm{min} / 1.73 \mathrm{~m}^{2}$ without subsequent CIN or those who developed CIN during index hospitalization, but had GFR within normal range at baseline $(6.32 \%$ vs. $2.90 \%, \mathrm{p}<0.05)$. The cumulative survival without stroke is shown in Figures 1 and 2 . The risk of stroke was especially high during the first month and first year after AMI $(0.54 \%$ and $2.47 \%$, respectively). Twenty-six patients $(78.8 \%)$ had ischemic strokes, whilst only $5(15.1 \%)$ strokes were hemorrhagic and $2(6.1 \%)$ undetermined.

\section{Independent predictors of stroke}

Patients who experienced stroke were more often female, with anterior location of MI, as well as with history of prior stroke. The baseline characteristics of both groups are summarized in Table 1 . According to the multivariate regression model, prior stroke (HR 6.85), female gender (HR 3.13) and anterior (HR 2.55) ST elevation myocardial

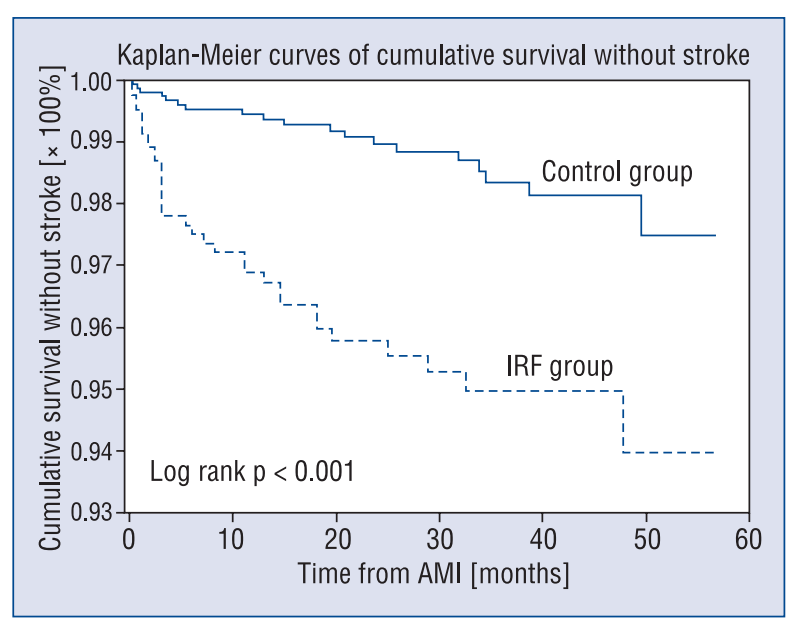

Figure 1. Kaplan-Meier curves of cumulative survival without stroke in the impaired renal function (IRF) population and the control group; $\mathrm{AMI}$ - acute myocardial infarction.

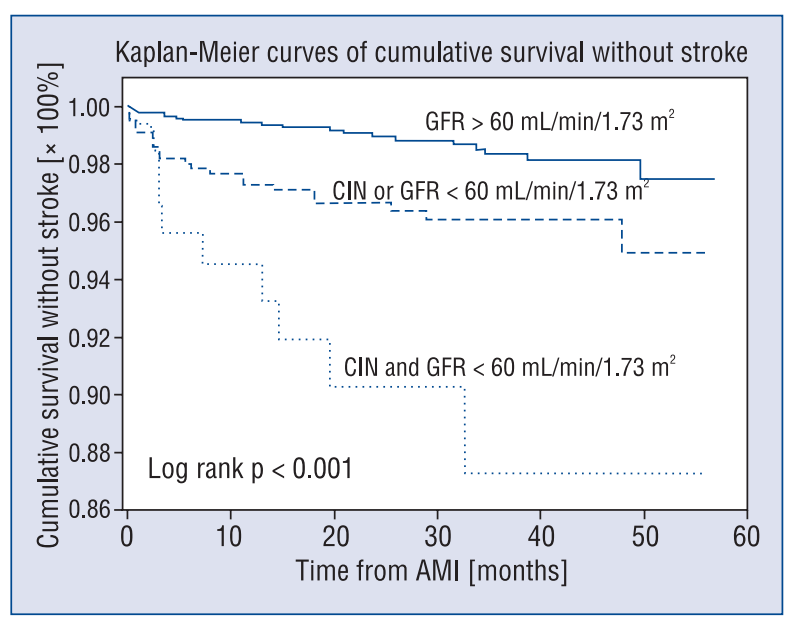

Figure 2. Cumulative probability of survival without stroke in particular groups of impaired renal function; AMI - acute myocardial infarction; GFR — glomerular filtration rate; $\mathrm{CIN}$ - contrast induced neprhropathy.

infarction (STEMI) were independent predictors of stroke in the analyzed population. However, the "conventional" stroke predictors were not found to be independent predictors of stroke in AMI patients treated invasively. The Cox proportional hazard model is shown in Table 2.

\section{Study outcomes}

The incidence of MACEs within 1-year and remote observation was significantly higher in patients from the stroke group. Compared with the control group these patients were at a higher risk of repeated AMI during 30-day, 12-month, as well as remote observation. Neither of the groups differed 
Table 1. The baseline characteristics of the study groups.

\begin{tabular}{|c|c|c|c|}
\hline Parameter & $\begin{array}{l}\text { Stroke group } \\
\quad(\mathrm{n}=33)\end{array}$ & $\begin{array}{l}\text { Non-stroke group } \\
(\mathrm{n}=900)\end{array}$ & $\mathbf{P}$ \\
\hline Female & $21(63.6 \%)$ & $345(38.3 \%)$ & $<0.05$ \\
\hline Age [years] & 65.9 & 66.0 & NS \\
\hline Diabetes mellitus & $17(51.5 \%)$ & $407(45.2 \%)$ & NS \\
\hline Hypertension & $23(69.7 \%)$ & $558(62.0 \%)$ & NS \\
\hline Atrial fibrillation & $4(12.1 \%)$ & $131(14.6 \%)$ & NS \\
\hline Hyperlipidemia & $18(54.5 \%)$ & $457(50.8 \%)$ & NS \\
\hline Smoking & $15(45.5 \%)$ & $418(46.4 \%)$ & NS \\
\hline Time from presentation to $\mathrm{PCl}[\mathrm{h}]$ & 6.6 & 7.2 & NS \\
\hline Prior stroke & $9(27.3 \%)$ & $55(6.1 \%)$ & $<0.001$ \\
\hline Prior AMI & $11(33.3 \%)$ & $214(23.8 \%)$ & NS \\
\hline Previous $\mathrm{PCl}$ & $3(9.1 \%)$ & $92(10.2 \%)$ & NS \\
\hline Previous CABG & $0(0 \%)$ & $31(3.4 \%)$ & NS \\
\hline STEMI anterior & $20(60.6 \%)$ & $344(38.2 \%)$ & $<0.05$ \\
\hline NSTEMI & $8(24.2 \%)$ & $203(22.6 \%)$ & NS \\
\hline $\mathrm{PCl}$ non-IRA & $8(24.2 \%)$ & $160(17.8 \%)$ & NS \\
\hline Hematocrit on admission & 39.5 & 40.8 & NS \\
\hline LVEF $[\%]$ & 42.3 & 40.7 & NS \\
\hline Thrombolysis & $1(3.0 \%)$ & $27(3.0 \%)$ & NS \\
\hline Glycoprotein Ilb/IIla receptor inhibitors & $8(24.2 \%)$ & $168(18.7 \%)$ & NS \\
\hline VKA at discharge & $1(3.0 \%)$ & $10(1.1 \%)$ & NS \\
\hline ASA at discharge & $32(97.0 \%)$ & $883(98.1 \%)$ & NS \\
\hline Thienopirydine at discharge & $32(97.0 \%)$ & $866(96.2 \%)$ & NS \\
\hline Beta-blockers at discharge & $30(90.9 \%)$ & $814(90.4 \%)$ & NS \\
\hline ACE-I at discharge & $29(87.9 \%)$ & $797(88.6 \%)$ & NS \\
\hline Statins at discharge & $29(87.9 \%)$ & $782(86.9 \%)$ & NS \\
\hline
\end{tabular}

ACE-I - angiotensin converting enzyme inhibitors; AMI - acute myocardial infarction; ASA — acetylsalicylic acid; CABG - coronary artery bypass grafting; GFR — glomerular filtration rate; IRA — infarct related artery; LVEF — left ventricular ejection fraction; NSTEMI - non-ST elevation myocardial infarction; PCI — percutaneous coronary intervention; STEMI — ST-elevation myocardial infarction; VKA — vitamin $\mathrm{K}$ antagonists

Table 2. Model of multivariate Cox regression.

\begin{tabular}{lccc}
\hline Parameter & Hazard ratio \pm 95\% confidence interval & Wald $\chi^{2}$ & P \\
\hline Female gender & $3.13(2.39-3.87)$ & 9.13 & $<0.05$ \\
Age $>$ 65 years & $1.03(0.12-1.94)$ & 0.01 & NS \\
Atrial fibrillation & $1.23(0.16-2.30)$ & 0.15 & NS \\
Anterior STEMI & $2.55(1.80-3.30)$ & 5.89 & $<0.05$ \\
Arterial hypertension & $0.75(0-1.53)$ & 0.50 & NS \\
Previous stroke & $6.85(6.03-7.67)$ & 20.70 & $<0.001$ \\
Diabetes mellitus & $1.13(0.42-1.84)$ & 0.11 & NS \\
LVEF $<35 \%$ & $0.92(0-1.85)$ & 0.03 & NS \\
VKA & $1.37(0.3-2.44)$ & 0.09 & NS \\
\hline
\end{tabular}

LVEF — left ventricular ejection fraction; STEMI — ST-elevation myocardial infarction; VKA — vitamin K antagonists

significantly with regard to short-term and 1-year mortality, however, remote mortality was significantly higher in the stroke group (45.5 vs. $25.1 \%$, $\mathrm{p}<0.05)$. Among 29 patients who survived stroke, $11(37.93 \%)$ died during long-term observation, and the median time interval between stroke and death was 16.6 (range: $0.7-38.2$ ) months. There were no significant differences between the studied groups in the need for repeated coronary revascularization, neither taking into account urgent/elective nor 
Table 3. Outcomes in patients with and without stroke after myocardial infarction.

\begin{tabular}{|c|c|c|c|}
\hline & Stroke group $(\mathrm{n}=33)$ & Non-stroke group $(n=900)$ & $\mathbf{P}$ \\
\hline \multicolumn{4}{|l|}{ 30-day outcome: } \\
\hline Myocardial infarction & $2(6.1 \%)$ & $10(1.1 \%)$ & $<0.05$ \\
\hline Elective $\mathrm{PCl}$ & $0(0 \%)$ & $9(1.0 \%)$ & NS \\
\hline Urgent $\mathrm{PCl}$ & $0(0 \%)$ & $5(0.6 \%)$ & NS \\
\hline Elective CABG & $0(0 \%)$ & $2(0.2 \%)$ & NS \\
\hline Urgent CABG & $0(0 \%)$ & $1(0.1 \%)$ & NS \\
\hline Mortality & $2(6.1 \%)$ & $139(15.4 \%)$ & NS \\
\hline MACE & $3(9.1 \%)$ & $160(17.8 \%)$ & NS \\
\hline \multicolumn{4}{|l|}{ One-year outcome: } \\
\hline Myocardial infarction & $11(33.3 \%)$ & $103(11.4 \%)$ & $<0.001$ \\
\hline Elective $\mathrm{PCl}$ & $5(15.2 \%)$ & $84(9.3 \%)$ & NS \\
\hline Urgent PCl & $3(9.1 \%)$ & $35(3.9 \%)$ & NS \\
\hline Elective CABG & $3(9.1 \%)$ & $46(5.1 \%)$ & NS \\
\hline Urgent CABG & $0(0 \%)$ & $1(0.1 \%)$ & NS \\
\hline Mortality & $9(27.3 \%)$ & $208(23.1 \%)$ & NS \\
\hline MACE & $22(66.7 \%)$ & $445(49.4 \%)$ & $<0.05$ \\
\hline \multicolumn{4}{|l|}{ Remote follow up: } \\
\hline Myocardial infarction & $11(33.3 \%)$ & $127(14.1 \%)$ & $<0.05$ \\
\hline Elective $\mathrm{PCl}$ & $6(18.2 \%)$ & $104(11.6 \%)$ & NS \\
\hline Urgent $\mathrm{PCl}$ & $3(9.1 \%)$ & $47(5.2 \%)$ & NS \\
\hline Elective CABG & $3(9.1 \%)$ & $53(5.9 \%)$ & NS \\
\hline Urgent CABG & $0(0 \%)$ & $2(0.2 \%)$ & NS \\
\hline Mortality & $15(45.5 \%)$ & $226(25.1 \%)$ & $<0.05$ \\
\hline MACE & $25(75.8 \%)$ & $503(55.9 \%)$ & $<0.05$ \\
\hline
\end{tabular}

CABG — coronary artery bypass grafting; MACE — major adverse cardiovascular event; PCl — percutaneous coronary intervention

percutaneous/surgical procedures. Additionally, patients with stroke developed more frequently end-stage renal failure requiring dialysis (12.12 vs. $2.89 \%, \mathrm{p}<0.05)$. The data on short- and long-term follow-ups are shown in Table 3.

\section{Discussion}

We have shown in our study that in AMI population the risk of stroke is 3 -fold higher in subjects with concomitant IRF compared to patients with normal renal function. This observation was concordant with previous reports, as IRF had been demonstrated to be a powerful risk factor for CV diseases, including stroke [3-5, 8]. However, contrary to prior studies our analysis was conducted on a homogenous AMI population treated invasively. This fact is an essential novelty of our study and may have had a significant impact on our results, as on the one hand PCI was proved to reduce the risk of stroke in AMI patients [15], and on the other hand contrast exposure may deteriorate renal function and increase $\mathrm{CV}$ risk associated with renal failure $[3-5,8]$.
Many distinct mechanisms have been proposed to account for increased CV risk in renal dysfunction. IRF is associated with oxidative stress, inflammation, a state of hypercoagulation with high levels of von Willebrand factor, fibrinogen, factors VII, VIII and IX, as well as enhanced thrombin generation and decrease in fibrinolytic activity, that promote plaques formation and deteriorate endothelial function $[16,17]$. It has been also suggested, that vascular inflammation may be an important mediator of both renal dysfunction and CV risk [8]. Additionally, higher blood pressure, dyslipidemia, microalbuminuria, homocysteinemia, as well as elevated plasma uric acid level accelerate atherosclerotic process in IRF population [3]. Contrast induced nephropathy developing in subjects with primarily decreased GFR may severely deteriorate renal function and amplify mechanisms mentioned-above leading to significant increase in $\mathrm{CV}$ risk.

Stroke, which occurred after AMI, independently of its timing, was a marker of heightened risk of death and recurrent MI during 1-year and remote follow-up. Our results are consistent with the findings from the OASIS study, which showed 
that a stroke subsequent to acute coronary syndrome was associated with significantly higher mortality at 6 -month follow-up (27 vs. $6.3 \%$, $\mathrm{p}<0.001$ ) [18]. Also other studies showed stroke population to be at a higher risk of $\mathrm{CV}$ adverse events $[19,20]$. Apart from its direct impact on mortality, the stroke may be a marker of severity of atherosclerotic process, endothelial dysfunction and hypercoagulation state. This hypothesis may partly explain a worse prognosis in patients with AMI who developed stroke.

We found previous stroke to be the strongest, independent predictor of next stroke in patients with AMI and concomitant IRF. It is in concordance with prior reports and is reasonable, as once recognized cerebrovascular disease is still present, it can progress and predisposes to a new cerebrovascular incident [3, 6-8]. Female gender, broadly postulated as a stroke predictor [6], was also independently associated with increased risk of stroke in AMI population with concomitant disturbed renal function.

Our analysis demonstrated that patients with anterior STEMI were at higher risk of subsequent stroke. The mechanisms underlying this predisposition are likely multifactorial. Anterior infarctions, usually resulting in larger necrotic area, may essentially predispose to heart failure and left ventricular thrombus formation [21, 22]. These factors might be also associated with increased predisposition to stroke in our group, as we observed significantly higher maximal values of MB isoenzyme of creatine kinase and lower LVEF in subjects with anterior STEMI than in patients with STEMI of other locations.

As opposed to previous studies [6, 9, 15, 19], we did not find $\mathrm{AF}$ to increase the risk of stroke in the studied population. One of possible explanations for it might be the fact, that over $95 \%$ of patients in our population were treated with dual anti-platelet therapy, whereas in prior studies monotherapy with acetylsalicylic acid (ASA) was mostly applied $[6,9,15,19]$. Our hypothesis is supported by the findings from the ACTIVE study, as according to them double anti-platelet therapy was proved to be more effective than ASA monotherapy in stroke prevention in AF population [23, 24]. Additionally, dual anti-platelet therapy was reported to be of equal effectiveness in stroke prophylaxis as oral VKA in patients with newly administered VKA therapy [23,24]. Therefore, combined therapy with ASA and thienopyridine may weaken the pro-thrombotic effect of AF, and hence may reduce the risk of ischemic stroke in the studied population.
The beneficial impact of renin-angiotensin system blockade on the risk of adverse CV events after AMI, especially in patients with chronic kidney disease, has been already demonstrated $[5,15$, 25]. In our population the rate of patients receiving angiotensin converting enzyme inhibitor (ACE-I) or angiotensin receptor blockers (ARB) exceededs $85 \%$ and was significantly higher than in prior studies. The high rate of ACE-I/ARB recipients and aggressive treatment of arterial hypertension might partially explain the lack of unfavorable effect of arterial hypertension on the risk of stroke in our study. In contrast to the majority of prior studies, heart failure was not associated significantly with the increased risk of stroke in the examined population $[6,15]$. Our findings were consistent with the results of the VALIANT study, which demonstrated that decreased LVEF was not predictive of stroke in high-risk patients after AMI [15]. We suppose that complex pharmacotherapy, by a variety of mechanisms, including: plaque stabilization, reduction in endothelial dysfunction, preventing ventricular remodeling and thrombus formation, could attenuate the negative effect of heart failure on the risk of stroke after MI [15, 24, 26].

Our results suggest that diabetes mellitus does not increase significantly the risk of stroke in AMI population with IRF. The strict glycemia control and intensive insulinotherapy administered in an early peri-infarct period could partially account for attenuation of risk associated with hyperglycemia status. The prothrombotic platelet and plasma properties in subjects with diabetes mellitus may be reduced thanks to achieving lower blood glucose levels that additionally could beneficially influence the lipid profile and endothelial function [27]. Our hypothesis is supported by findings from the DIGAMI II study, as stricter diabetes control was demonstrated to beneficially influence the risk of $\mathrm{CV}$ adverse events, including stroke, in subjects after AMI [28].

We suppose that in patients with AMI and concomitant impaired renal function early invasive treatment and routine complex pharmacotherapy may reduce the risk of stroke, as well as attenuate the significance of "conventional" stroke predictors, and simultaneously increase the role of other risk factors, nevertheless, further research is needed.

\section{Limitations of the study}

All analyses were based on observational data, with all the drawbacks associated with data collected this way. Because of the relatively small population and low number of neurological incidents during follow-up, some important parameters, 
influencing the risk of stroke, might have been underestimated. Therefore, our results should be considered hypothesis generating data.

\section{Conclusions}

The patients treated invasively in the acute phase of MI, with concomitant impaired renal function, are at higher risk of stroke, especially within the early post-AMI period. The population with primarily decreased GFR and overlapping contrast induced nephropathy is the group of extremely high CV risk. The independent risk factors for stroke in patients with IRF and AMI treated with PCI are only partially consistent with commonly recognized stroke predictors. The stroke ought to be considered a significant marker of worse prognosis after MI.

\section{Conflict of interest: none declared}

\section{References}

1. Go AS, Yang J, Ackerson LM et al. Hemoglobin level, chronic kidney disease, and the risks of death and hospitalization in adults with chronic heart failure: the Anemia in Chronic Heart Failure: Outcomes and Resource Utilization (ANCHOR) Study. Circulation, 2006; 113: 271-223.

2. Ezekowitz J, McAlister FA, Humphries KH et al.; APPROACH Investigators. The association among renal insufficiency, pharmacotherapy and outcomes in 6,427 patients with heart failure and coronary artery disease. J Am Coll Cardiol, 2004; 44: 1587-1592.

3. Anavekar NS, McMurray JJ, Velazquez EJ et al. Relation between renal dysfunction and cardiovascular outcomes after myocardial infarction. N Engl J Med, 2004; 35: 1285-1295.

4. Gibson CM, Pinto DS, Murphy SA et al; TIMI Study Group (2003) Association of creatinine and creatinine clearance on presentation in acute myocardial infarction with subsequent mortality. J Am Coll Cardiol, 2003; 42: 1535-1543.

5. Tokmakova MP, Skali H, Kenchaiah S et al. Chronic kidney disease, cardiovascular risk, and response to angiotensin-converting enzyme inhibition after myocardial infarction: the Survival And Ventricular Enlargement (SAVE) study. Circulation, 2004; 110: 3667-3673.

6. Witt BJ, Ballman KV, Brown RD Jr, Meverden RA, Jacobsen SJ, Roger VL. The incidence of stroke after myocardial infarction: A meta-analysis. Am J Med, 2006; 119: 354.e1-9.

7. Herlitz J, Holm J, Peterson M, Karlson BW, Evander MH, Erhardt L; LoWASA Study Group. Factors associated with development of stroke long-term after myocardial infarction: experiences from the LoWASA trial. J Intern Med, 2005; 257: 201-207.

8. Mielniczuk LM, Pfeffer MA, Lewis EF et al. Estimated glomerular filtration rate, inflammation, and cardiovascular events after an acute coronary syndrome. Am Heart J, 2008; 155: 725-731.

9. Mooe T, Eriksson P, Stegmayr B. Ischemic stroke after acute myocardial infarction. A population-based study. Stroke, 1997; 28: 762-767.

10. Loh E, Sutton MS, Wun CC et al. Ventricular dysfunction and the risk of stroke after myocardial infarction. N Engl J Med, 1997; 336: 251-257.

11. Bertrand ME, Simoons ML, Fox KA et al. Management of acute coronary syndromes in patients presenting without persistent ST-segment elevation. Eur Heart J, 2002; 23: 1809-1840.

12. Van de Werf F, Ardissino D, Betriu A et al. Management of acute myocardial infarction in patients presenting with ST-segment elevation. The Task Force on the Management of Acute Myocardial Infarction of the European Society of Cardiology. Eur Heart J, 2003; 24: 28-66.

13. Silber S, Albertsson P, Avilés FF et al. Guidelines for percutaneous coronary interventions. The Task Force for Percutaneous Coronary Interventions of the European Society of Cardiology. Eur Heart J, 2005; 26: 804-847.

14. Levey AS, Coresh J, Balk E et al.; National Kidney Foundation. National Kidney Foundation practice guidelines for chronic kidney disease: evaluation, classification, and stratification. Ann Intern Med, 2003; 139: 137-147.

15. Sampson UK, Pfeffer MA, McMurray JJ, Lokhnygina Y, White HD, Solomon SD; VALIANT Trial Investigators. Predictors of stroke in high-risk patients after acute myocardial infarction: insights from the VALIANT Trial. Eur Heart J, 2007; 28: 685-691.

16. Gibson CM, Dumaine RL, Gelfand EV et al; TIMI Study Group. Association of glomerular filtration rate on presentation with subsequent mortality in non-ST-segment elevation acute coronary syndrome: Observations in 13307 patients in five TIMI trials. Eur Heart J, 2004; 25: 1998-2005.

17. Stam F, van Guldener C, Schalkwijk CG, ter Wee PM, Donker AJ, Stehouwer CD. Impaired renal function is associated with markers of endothelial dysfunction and increased inflammatory activity. Nephrol Dial Transplant, 2003; 18: 892-898.

18. Cronin L, Mehta SR, Zhao F et al. Stroke in relation to cardiac procedures in patients with non-ST-elevation acute coronary syndrome: a study involving > 18000 patients. Circulation, 2001; 104: 269-274.

19. Tanne D, Goldbourt U, Zion M, Reicher-Reiss H, Kaplinsky E, Behar S. Frequency and prognosis of stroke/TIA among 4808 survivors of acute myocardial infarction. The SPRINT Study Group. Stroke, 1993; 24: 1490-1495.

20. Szummer KE, Solomon SD, Velazquez EJ et al.; VALIANT Registry. Heart failure on admission and the risk of stroke following acute myocardial infarction: The VALIANT registry. Eur Heart J, 2005; 26: 2114-2119.

21. Lamas GA, Vaughan DE, Pfeffer MA. Left ventricular thrombus formation after first anterior wall acute myocardial infarction. Am J Cardiol, 1988; 62: 31-35.

22. Vaitkus PT, Barnathan ES. Embolic potential, prevention and management of mural thrombus complicating anterior myocardial infarction: a meta-analysis. J Am Coll Cardiol, 1993; 22: 1004-1009.

23. Healey JS, Hart RG, Pogue J et al. Risks and benefits of oral anticoagulation compared with clopidogrel plus aspirin in patients with atrial fibrillation according to stroke risk: the atrial fibrillation clopidogrel trial with irbesartan for prevention of vascular events (ACTIVE-W). Stroke, 2008; 39: 1482-1486.

24. Connolly SJ, Pogue J, Hart RG et al.; ACTIVE Investigators. Effect of clopidogrel added to aspirin in patients with atrial fibrillation. N Engl J Med, 2009; 360: 2066-2078.

25. Yusuf S, Sleight P, Pogue J, Bosch J, Davies R, Dagenais G. Effects of an angiotensin-converting-enzyme inhibitor, ramipril, on cardiovascular events in high-risk patients. The Heart Outcomes Prevention Evaluation Study Investigators. N Engl J Med, 2000; 342: 145-153.

26. Blauw GJ, Lagaay AM, Smelt AH, Westendorp RG. Stroke, statins, and cholesterol. A meta-analysis of randomized, placebo-controlled, double-blind trials with HMG-CoA reductase inhibitors. Stroke, 1997; 28: 946-950.

27. Malmberg K, Rydén L, Hamsten A, Herlitz J, Waldenström A, Wedel H. Effects of insulin treatment on cause-specific one-year mortality and morbidity in diabetic patients with acute myocardial infarction. DIGAMI Study Group. Diabetes Insulin-Glucose in Acute Myocardial Infarction. Eur Heart J, 1996; 17: 1337-1344.

28. Malmberg K, Rydén L, Wedel H et al.; DIGAMI 2 Investigators. Intense metabolic control by means of insulin in patients with diabetes mellitus and acute myocardial infarction (DIGAMI 2): effects on mortality and morbidity. Eur Heart J, 2005; 26: 650-661. 